\title{
The new ISPD peritonitis guideline
}

\author{
Cheuk Chun Szeto
}

\begin{abstract}
Peritoneal dialysis (PD)-related infection encompasses PD-related peritonitis and catheter-related infections, and the latter is used as the collective term to describe exit site infection (ESI) and tunnel infection. Despite of the advances in technology and antibiotic therapy, PD-related infections remain common and serious complications of PD. Since 2016, the International Society for Peritoneal Dialysis (ISPD) has published revised guidelines on the treatment and prevention of peritonitis and catheter-related infections. The two sets of guideline include evidence-based review and opinion-based recommendations. In this article, I review the key points and major changes in the 2016/17 version of ISPD recommendations. The recent literature that resulted in these changes will be highlighted. Problems related to implementation of these recommendations in different countries will also be discussed.
\end{abstract}

Keywords: Renal failure, Infection, Microbology, Survival

\section{Background}

Peritonitis is a common and serious complication of peritoneal dialysis (PD). Recommendations for the treatment and prevention of PD-related peritonitis, and latter those for catheter-related infections, under the auspices of the International Society for Peritoneal Dialysis (ISPD) were first published in 1987 and revised in 1989, 1993, 1996, 2000, 2005, and 2010 [1-8]. In the previous version published in 2010 to 2011, a set of guideline was published for the treatment of peritonitis and catheterrelated infections [7], and a separate one for their prevention [8]. In the latest version, a single set of recommendations was provided for the treatment and prevention of PD-related peritonitis [9], and another for catheter-related infections [10]. In this article, we will focus on the new changes and key messages of the new ISPD guideline on the treatment and prevention of peritonitis [9]. Unless otherwise specified, all recommendation statements in this article are cited from this document [9].

\section{Monitoring and reporting}

The latest ISPD recommendation states that every PD center should monitor, at least on a yearly basis, the incidence of peritonitis [9]. Notably, peritonitis rate should

Correspondence: ccszeto@cuhk.edu.hk

From Carol and Richard Yu of Peritoneal Dialysis Research Centre,

Department of Medicine and Therapeutics, Prince of Wales Hospital, The Chinese University of Hong Kong, Shatin, Hong Kong SAR, China.

Department of Medicine and Therapeutics, Prince of Wales Hospital, The Chinese University of Hong Kong, Shatin, Hong Kong be reported as number of episodes per patient-year rather than as one episode per number of patient-month of treatment, which used to be commonly used. Organism-specific peritonitis rates should be reported, also as absolute rates. The recommendation does not specify which organisms should be monitored, but it seems reasonable to focus on the ones that are common in individual PD unit. Other parameters to be monitored include the percentage of patients per year who are peritonitis free, the median peritonitis rate for the program (determined by calculating the peritonitis rate for each patient, and then obtain the median of these rates), and the antimicrobial susceptibilities of the infecting organisms. In addition, sampling and culture methods of PD effluent should be reviewed and improved if more than $15 \%$ of peritonitis episodes are culture negative in a PD center.

\section{Diagnosis and empirical treatment}

There is no major change in the diagnosis or empirical treatment of PD-related peritonitis. As in the previous versions, peritonitis should still be diagnosed when at least two of the following are present: (i) clinical features consistent with peritonitis, i.e., abdominal pain and/or cloudy dialysis effluent; (ii) dialysis effluent white cell count above $100 / \mu \mathrm{L}$ or with over $50 \%$ of polymorphonulcear leukocyte in the differential count; and (iii) identification of infective organisms from by dialysis effluent Gram statin or culture [9]. The important message for practicing nephrologists is PD patients presenting with cloudy PD effluent should be presumed to have 
peritonitis and treated as such (after appropriate workup and specimen collection) until the diagnosis can be confirmed or excluded. PD effluent should be tested for cell count, differential, Gram stain, and culture whenever peritonitis is suspected, and blood-culture bottle is the preferred technique for bacterial culture of PD effluent. The latest guideline concluded that there is insufficient evidence to currently support the use of novel techniques for the diagnosis of peritonitis [9].

As emphasized repeatedly in recent three versions of the guideline $[6,7,9]$, empirical antibiotic therapy should be initiated as soon as possible after appropriate microbiological specimens have been obtained. The recommended empirical antibiotic regimens have not changed in the latest version and should be center-specific and cover both Gram-positive and Gram-negative organisms [9]. Gram-positive organisms could be covered by vancomycin or a first-generation cephalosporin; Gram-negative organisms could be covered by a thirdgeneration cephalosporin or an aminoglycoside (e.g., gentamicin).

\section{Practical issues of peritonitis treatment}

The presence of residual renal function substantially affects intra-peritoneal drug levels in both continuous ambulatory peritoneal dialysis (CAPD) and machine-assisted automated peritoneal dialysis (APD), and affects antibiotic levels in both continuous and intermittent dosing, but may be relatively more important with intermittent dosing because antibiotic is not replenished with each exchange. For patients with substantial residual renal function (e.g., daily urine output over $100 \mathrm{~mL}$ ), the previous version of the ISPD guideline recommends empirically increasing antibiotic dose by $25 \%$ [7]. This recommendation is removed from the latest version [9] in order to reflect the paucity of published data in this area.

As emphasized in the previous guideline, intraperitoneal (IP) antibiotics is the preferred route of administration unless the patient has features of systemic sepsis. Although the recommended dosage for continuous administration was described in the text, IP aminoglycoside should preferably be administered as daily intermittent dosing [9]. Treatment of IP aminoglycoside for over 3 weeks should be avoided because of the very high risk of ototoxicity revealed by recent literature [11]. IP vancomycin should also be administered intermittently. The latest guideline further suggests that the serum trough vancomycin level should be kept above $15 \mu \mathrm{g} / \mathrm{mL}$. The indication or frequency of vancomycin level monitoring, however, is not mentioned. We believe it is valuable to check vancomycin level in patients with substantial residual renal function, 3 to 4 days after the first dose of IP vancomycin. Unlike aminoglycosides and vancomycin, IP cephalosporin could be administered either continuously (in each exchange) or on a daily intermittent basis.

Although the stability of antibiotics in PD solutions has long been a theoretical concern, recent evidence suggests that all first-line IP antibiotics are stable for routine clinical use, including pre-injection by helpers $[12,13]$. However, when vancomycin and gentamicin are used in combination, they should preferably be injected to different bags of PD solution [13], and vancomycin should not be given into amino-acid based solution [14]. The latest version of the guideline also provide an exhaustive list of antibiotics suitable for IP administration, together with their recommended dosage and supporting literature [9]. These agents include cefepime, cefoperazone, piperacillin/tazobactam, ampicillin/sulbactam (but not amoxicillin/clavulanate), daptomycin, quinupristin/ dalfopristin, teicoplanin, imipenem/cilastatin, and meropenam. Unlike usually first-line antibiotics, however, ampicillin and linezolid should not be given IP because of chemical instability in PD solutions [15]. A number of other antibiotics could be used systemically as alternative or second-line agents, including ciprofloxacin, levofloxacin, moxifloxacin, ertapenem, colistin, rifampicin, and trimethoprim/sulfamethoxazole. Most antifungal agents should also be given systemically.

For APD patients with peritonitis, the guideline provides several options. In essence, antibiotics could be administered IP into the bag of day-time long dwell PD solution, or the patient could be temporarily converted to CAPD. The latter option, however, is not always feasible. APD patients may not have been taught on the technique of CAPD exchange, and there are probably no appropriate PD solutions at home for the treatment [16].

As in the previous versions, the latest guideline describes several auxiliary measures. Rapid peritoneal flushing is possible but has doubtful benefit. Intra-peritoneal heparin is recommended to prevent fibrin formation in the PD fluid. Secondary prophylaxis of fungal peritonitis by nystatin or fluconazole should always be used.

\section{Subsequent management}

Once culture results and sensitivities are known, antibiotic therapy should be adjusted to narrow spectrum agents as appropriate. Coagulase-negative staphylococci peritonitis should generally be treated with IP firstgeneration cephalosporins or vancomycin, according to antimicrobial susceptibility, for 2 weeks. Staphylococcus aureus peritonitis should be treated for 3 weeks, probably with a short course of oral rifampicin to eradicate concomitant carrier state as well as adherent or intracellular organisms. Streptococcal peritonitis should be treated for 2 weeks, while corynebacterial peritonitis should be treated for 3 weeks. 
The latest guideline highlights the unique features of peritonitis caused by Enterococcus species. In essence, enterococcal peritonitis should be treated with IP vancomycin for 3 weeks, and IP aminoglycoside should be added for severe cases. For peritonitis due to vancomycin-resistant enterococcus (VRE), treatment for 3 weeks with IP ampicillin if the organism is susceptible, or with alternative antibiotics (linezolid, quinupristin/ dalfopristin, daptomycin or teicoplanin, based on antimicrobial susceptibilities) if the organism is ampicillinresistant. As mentioned above, IP ampicillin may not be chemically stable and its efficacy has been questioned. Our recent data suggests that oral amoxicillin is an effective alternative in this scenario (Szeto CC, manuscript under preparation).

As in the previous guidelines, Pseudomonas peritonitis should be treated with two antibiotics with different mechanisms of action (e.g., IP gentamicin or oral ciprofloxacin with IP ceftazidime or cefepime) for 3 weeks. The latest version emphasizes that Pseudomonas peritonitis with concomitant exit site and tunnel infection should have catheter removal [9]. Unlike the previous recommendations, non-Pseudomonas Gram-negative peritonitis should now be treated for at least 3 weeks [9]. If multiple enteric organisms (multiple Gramnegative or mixed Gram-negative/Gram-positive organisms) are grown from PD effluent, surgical evaluation should be obtained immediately when there is no prompt clinical response, and that the patient should be treated with metronidazole in conjunction with IP vancomycin (to cover Enterococcal species) and either an IP aminoglycoside or IP ceftazidime for a minimum period of 3 weeks. If multiple Gram-positive organisms are grown from PD effluent, the patient should be treated with effective antibiotics for 3 weeks.

When no bacteria are identified from the PD effluent culture by day 3 , a repeat dialysis effluent WBC count with differential and bacterial culture should be performed. If the culture-negative peritonitis is resolving at day 3, aminoglycoside should be discontinued, and Gram-positive coverage (i.e., first-generation cephalosporin or vancomycin) should be continued for a total of 2 weeks. However, the guideline does not specify whether ceftazidime should be stopped when it is used as the empirical Gram-negative coverage. We believe such decision should be based on the local prevalence of Gram-negative peritonitis. If the culture-negative peritonitis is not resolving at day 3 , special culture techniques should be considered for isolation of unusual organisms.

If fungus is identified from the PD effluent, the guideline emphasizes immediate catheter removal. Treatment with an appropriate anti-fungal agent should be continued for at least 2 weeks after catheter removal. Unlike bacterial peritonitis, IP anti-fungal agent has no preferential role over systemic therapy. Although amphotericin is effective, adverse effects are common and serious. The latest guideline subtly suggests the use of echinocandins (e.g., caspofungin) for Aspergillus and nonalbicans Candida species, and second-generation azoles (e.g., posaconazole and voriconazole) for filamentous fungi. However, it should be noted that intravenous voriconazole contains cyclodextrin as solvent, which may accumulate in renal failure patients and cause neurotoxicity.

The latest guideline gives very clear cut indications of catheter removal: refractory peritonitis, relapsing peritonitis, refractory exit site and tunnel infection, and fungal peritonitis. Catheter removal may also be considered for repeat peritonitis, mycobacterial peritonitis, and peritonitis caused by multiple enteric organisms. Although there is no major change in the terminology of recurrent, relapsing, and repeat peritonitis episodes, refractory peritonitis episodes are now defined as failure of the effluent to clear after 5 days (not $96 \mathrm{~h}$ as used previously) of appropriate antibiotics [9]. After catheter removal, systemic antibiotics should be continued for at least 2 weeks. It is appropriate to consider return to PD for many patients who have had their catheter removed for refractory, relapsing, or fungal peritonitis. If re-insertion of a new catheter is attempted, it should be performed at least 2 weeks after catheter removal and complete resolution of peritoneal symptoms.

\section{Secondary prevention}

As mentioned above, fungal prophylaxis during antibiotic therapy may prevent secondary Candida peritonitis, at least in programs that have high rates of fungal peritonitis. The latest guideline actually states that antifungal prophylaxis should be given when PD patients receive antibiotic therapy (i.e., not only for the treatment of peritonitis) [9]. However, many centers only restrict the use of anti-fungal prophylaxis during peritonitis treatment. Possible options include nystatin and fluconazole. Although nystatin is generally preferred, it is not available in some countries, and the drug comes in variable formulations such as tablet and mouth wash solution, and their efficacy has not been compared. Fluconazole is effective but the literature support is less strong, and there are potential problems of its extensive use, including drug interactions and emergence of resistant strains.

The latest guideline highlights the importance of performing a root cause analysis for each peritonitis episode to determine the etiology of the episode, and, whenever possible, further intervention should be directed against any reversible risk factor to prevent another episode. The guideline specifically mentioned that identification of etiology may involve review of the patient's technique, and, if necessary, retraining should be performed. 


\section{Primary prevention}

Although many modifiable risk factors have been identified for PD-related peritonitis [17], only few of them have been showed to be effective therapeutic targets. The latest guideline makes a few specific recommendations on evidence-based strategies for the prevention of peritonitis. First, systemic prophylactic antibiotics should be administered immediately prior to catheter insertion, which is highly effective in reducing the incidence of early peritonitis after catheter insertion, although the evidence for the prevention of catheter-related infections (i.e., exit site or tunnel infections) is less solid $[9,10]$. The choice of antibiotics is less important; cefazolin, vancomycin, or other beta lactam group antibiotics all seem reasonable choices. In addition to before PD catheter insertion, antibiotic prophylaxis should also be given before other invasive procedures (e.g., dental, colonoscopy, polypectomy, hysteroscopy, and cholecystectomy) and after wet contamination.

Second, disconnect systems with a "flush before fill" design should be used for CAPD. Different disconnect systems (e.g., double-bag versus Y-set systems) have little difference. In addition, the guideline makes no specific recommendation on the choice of $\mathrm{PD}$ solution. The evidence of biocompatible solution on the risk of peritonitis is inconclusive. Other measures related to the catheter, including catheter design, position of the exit site, method of catheter insertion (e.g., laparoscopy versus laparotomy), and other novel catheter techniques, show no benefit by meta-analysis $[18,19]$. For the care of catheter exit site, the guideline recommends daily topical application of antibiotic (mupirocin or gentamicin) cream or ointment. Any active exit site or catheter tunnel infection should be promptly treatment.

The latest guideline also emphasizes the adherence of the latest ISPD recommendations for teaching PD patients and their caregivers [20]. PD training should be conducted by nursing staff with the appropriate qualifications and experience. Unfortunately, optimal treatment program is vaguely defined and actually highly variable in the literature. In addition to meticulous patient training, each PD center should have a continuous quality improvement (CQI) program in place. The latest guideline further specifies that multidisciplinary teams running CQI programs should meet and review their units' performance metrics regularly. The guideline also suggests a list of indications for re-training: following prolonged hospitalization; following peritonitis or catheter infection; following change in dexterity, vision, or mental acuity; following change to another supplier or a different type of connection; and following other interruption in PD (e.g., period of time on hemodialysis). Published evidence to support this recommendation, however, is limited.

\section{Conclusions}

In essence, the latest guideline summarizes a number of measures with proved efficacy for the prevention of peritonitis: antibiotic prophylaxis before catheter insertion, the application of disconnect PD system, and regular use of antibiotic cream or ointment for exit site care. On the other hand, the benefit of other novel exit site remedies, biocompatible PD solution, and patient retraining program remain uncertain.

As to the treatment of PD peritonitis, the new guideline emphasizes the standardization of reporting by the absolute peritonitis rate. Under the current recommendations, most peritonitis should be treated for 3 weeks, except those caused by coagulase-negative staphylococcal species or Streptococcus. Prolonged treatment with gentamicin should be avoided. Blood vancomycin level should be monitored and dosage regimen adjusted accordingly. Catheter removal should be performed when there is no response to antibiotics after 5 days of treatment. Furthermore, the latest guideline includes extensive tables that describe the recommended dosage for individual antibiotics and should be frequently referred to during patient treatment.

A number of areas remain unclear under the latest guideline and deserve further studies. Notably, dosage adjustment for patients with substantial residual renal function, the management of patients on machineassisted PD, the use of anti-fungal prophylaxis during antibiotics therapy for indications other than peritonitis, the optimal method and frequency of patient training and re-training, and strategies for the prevention of relapsing and recurrent peritonitis episodes are all important research topics.

\section{Acknowledgements}

Not applicable.

\section{Funding}

This study was supported by the Chinese University of Hong Kong (CUHK) research account 6901031

Availability of data and materials

Data sharing is not applicable.

Ethics approval and consent to participate

Not applicable.

Consent for publication

Not applicable.

Competing interests

The author declares that he has no competing interests.

\section{Publisher's Note}

Springer Nature remains neutral with regard to jurisdictional claims in published maps and institutional affiliations. 
Received: 30 October 2017 Accepted: 25 January 2018

\section{Published online: 02 February 2018}

\section{References}

1. Keane WF, Everett ED, Fine RN, Golper TA, Vas S, Peterson PK. CAPD related peritonitis management and antibiotic therapy recommendations: Travenol Peritonitis Management Advisory Committee. Perit Dial Int. 1987;7:55-78.

2. Keane WF, Everett ED, Fine RN, Golper TA, Vas S, Peterson PK, Gokal R, Matzke GR. Continuous ambulatory peritoneal dialysis (CAPD) peritonitis treatment recommendations: 1989 update. The Ad Hoc Advisory Committee on Peritonitis Management. Perit Dial Int. 1989;9:247-56.

3. Keane WF, Everett ED, Golper TA, Gokal R, Halstenson C, Kawaguchi Y, Riella M, Vas S, Verbrugh HA. Peritoneal dialysis-related peritonitis treatment recommendations. 1993 update. The Ad Hoc Advisory Committee on Peritonitis Management. International Society for Peritoneal Dialysis. Perit Dial Int. 1993;13:14-28.

4. Keane WF, Alexander SR, Bailie GR, Boeschoten E, Gokal R, Golper TA, Holmes CJ, Huang CC, Kawaguchi Y, Piraino B, Riella M, Schaefer F, Vas S. Peritoneal dialysis-related peritonitis treatment recommendations: 1996 update. Perit Dial Int. 1996;16:557-73.

5. Keane WF, Bailie GR, Boeschoten E, Gokal R, Golper TA, Holmes CJ, Kawaguchi Y, Piraino B, Riella M, Vas S. Adult peritoneal dialysis-related peritonitis treatment recommendations: 2000 update. Perit Dial Int. 2000;20:396-411.

6. Piraino B, Bailie GR, Bernardini J, Boeschoten E, Gupta A, Holmes C, Kuijper EJ, Li PK, Lye WC, Mujais S, Paterson DL, Fontan MP, Ramos A, Schaefer F, Uttley L. ISPD Ad Hoc Advisory Committee. Peritoneal dialysis-related infections recommendations: 2005 update. Perit Dial Int 2005; 25: 107-131.

7. Li PK, Szeto CC, Piraino B, Bernardini J, Figuiredo AE, Gupta A, Johnson DW, Kuijper EJ, Lye WC, Salzer W, Schaefer F, Struijk DG. Peritoneal dialysis-related infections recommendations: 2010 update. Perit Dial Int. 2010;30:393-423.

8. Piraino B, Bernardini J, Brown E, Figueiredo A, Johnson DW, Lye WC, Price V, Ramalasmi S, Szeto CC. ISPD position statement on reducing the risks of peritoneal dialysis-related infections. Perit Dial Int. 2011;31:614-30.

9. Li PK, Szeto CC, Piraino B, de Arteaga J, Fan S, Figueiredo AE, Fish DN, Goffin E, Kim YL, Salzer W, Struijk DG, Teitelbaum I, Johnson DW. ISPD peritonitis recommendations: 2016 update on prevention and treatment. Perit Dial Int. 2016:36:481-508.

10. Szeto CC, Li PK, Johnson DW, Bernardini J, Dong J, Figueiredo AE, Ito Y, Kazancioglu R, Moraes T, van Esch S, Brown EA. ISPD catheter-related infections recommendations: 2017 update. Perit Dial Int. 2017;37:141-54.

11. Gendeh BS, Said H, Gibb AG, Aziz NS, Kong N, Zahir ZM. Gentamicin ototoxicity in continuous ambulatory peritoneal dialysis. J Laryngol Otol. 1993;107:681-5.

12. Bailie GR, Kane MP. Stability of drug additives to peritoneal dialysate. Perit Dial Int. 1995;15:328-35.

13. Patel RP, Shastri MD, Bakkari M, Wanandy T, Jose MD. Stability of the combination of ceftazidime and cephazolin in icodextrin or $\mathrm{pH}$ neutral peritoneal dialysis solution. Perit Dial Int. 2014;34:212-8.

14. Tobudic S, Poeppl W, Kratzer C, Vychytil A, Burgmann H. Comparative in vitro antimicrobial activity of vancomycin, teicoplanin, daptomycin and ceftobiprole in four different peritoneal dialysis fluids. Eur J Clin Microbiol Infect Dis. 2012;31:1327-34.

15. Kussmann M, Schuster L, Zeitlinger M, Pichler P, Reznicek G, Wiesholzer $M$ Burgmann H, Poeppl W. The influence of different peritoneal dialysis fluids on the in vitro activity of ampicillin, daptomycin, and linezolid against Enterococcus faecalis. Eur J Clin Microbiol Infect Dis. 2015;34:2257-63.

16. Szeto CC. Peritoneal dialysis-related infection in the older population. Perit Dial Int. 2015;35:659-62.

17. Cho Y, Johnson DW. Peritoneal dialysis-related peritonitis: towards improving evidence, practices, and outcomes. Am J Kidney Dis. 2014;64:278-89.

18. Strippoli GF, Tong A, Johnson D, Schena FP, Craig JC. Catheter-related interventions to prevent peritonitis in peritoneal dialysis: a systematic review of randomized, controlled trials. J Am Soc Nephrol. 2004;15:2735-46.

19. Hagen SM, Lafranca JA, IJzermans JN, Dor FJ. A systematic review and metaanalysis of the influence of peritoneal dialysis catheter type on complication rate and catheter survival. Kidney Int. 2014;85:920-32.

20. Figueiredo AE, Bernardini J, Bowes E, Hiramatsu M, Price V, Su C, Walker R, Brunier G. A syllabus for teaching peritoneal dialysis to patients and caregivers. Perit Dial Int. 2016;36:592-605.

\section{Submit your next manuscript to BioMed Central and we will help you at every step:}

- We accept pre-submission inquiries

- Our selector tool helps you to find the most relevant journal

- We provide round the clock customer support

- Convenient online submission

- Thorough peer review

- Inclusion in PubMed and all major indexing services

- Maximum visibility for your research

Submit your manuscript at www.biomedcentral.com/submit
Biomed Central 